\title{
$\eta-{ }^{4}$ He Bound States in the Skyrme Model
}

\author{
N. N. Scoccola ${ }^{1}$ !' and D.O. Riska ${ }^{2}$ \\ ${ }^{1}$ Physics Department, CNEA, Av. Libertador 8250, (1429) Buenos Aires, Argentina. \\ ${ }^{2}$ Department of Physics, University of Helsinki, 00014 Finland.
}

July 1998

\begin{abstract}
The rational map ansatz for light nuclei in the Skyrme model is shown to imply the existence of an $\eta-{ }^{4} \mathrm{He}$ bound state, with a binding energy of $\sim 30 \mathrm{KeV}$.
\end{abstract}

PACS: 12.39Dc, 14.40.Aq, 27.10.+h

Keywords: Rational maps, multiskyrmions, eta-nucleus resonances.

Considerable progress has been made recently in the development of calculational methods for realistic applications of Skyrme's topological soliton model for baryons [河 to the structure of light nuclei [2, 3, 因, 河. This significantly extends the applicability of the Skyrme model, which hitherto had largely been restricted to the structure of baryons [6, [7. The "rational map" method [4] in particular facilitates the development of simple yet realistic approximations to the full Skyrme model solution for spatially extended baryonic systems with $\mathrm{A}>1$. A demonstration of the utility of this method has recently been given in the study of strange multiskyrmions in ref. [8].

We here apply the rational map ansatz to show that $\eta$ mesons are very likely to form bound states with $\alpha$-particles. This gives further support to the long standing expectation that such should be formed [9, 10], and which was reinforced by the recent observation that the $\eta-d$ scattering length is $\sim 4 \mathrm{fm}$, i.e. almost 6 times larger than the $\eta$-nucleon scattering length [11. The present experimental results are consistent with the existence of a lightly bound state of $\eta$-mesons and $\alpha$-particles, although the results remain somewhat inconclusive [12, 13.

Theoretically the question of $\eta-{ }^{4} \mathrm{He}$ bound states is related to the question of the interaction between heavy quarkonia and nuclei [14, 15]. This issue has been investigated within the framework of the Skyrme model, which allows a coherent treatment of the $\eta, \eta_{c}$ and $\eta_{b}$ mesons with the same Lagrangian density in ref. [16]. The conclusion reached was that even though the $\eta$ meson does not form bound states with nucleons, the much heavier $\eta_{c}$ and $\eta_{b}$ mesons are very likely to form such. Thus e.g. the $c \bar{c}$ analog of the $\eta N 1 / 2^{-}$resonance $N(1535)$ predicted at $\simeq 2820 \mathrm{MeV}$ would be stable against strong decay.

\footnotetext{
${ }^{\dagger}$ Fellow of the CONICET, Argentina.
} 
The Skyrme model has the conceptual advantage over more phenomenological approaches to the coupling of $\eta$ mesons and nuclei in that the same Lagrangian density with one set of parameter values applies to several nuclear systems. The model itself represents an effective representation of the large $N_{C}$ limit of QCD [17].

To describe the coupling between the $\eta$-meson and baryonic matter we employ Pari's version of the Skyrme model [18]:

$$
\mathcal{L}=-\frac{f_{\pi}^{2}}{4} \operatorname{tr}\left\{L_{\mu} L^{\mu}\right\}+\frac{x}{32 e^{2}} \operatorname{tr}\left\{\left[L_{\mu}, L_{\nu}\right]^{2}\right\}+\frac{1-x}{16 e^{2}}\left[\left(\operatorname{tr}\left\{L_{\mu} L_{\nu}\right\}\right)^{2}-\left(\operatorname{tr}\left\{L_{\mu} L_{\mu}\right\}\right)^{2}\right]+\mathcal{L}_{S B}
$$

Here $L_{\mu} \equiv U^{\dagger} \partial_{\mu} U$, where $U$ is the $S U(3)$ extension of the skyrmion field, $f_{\pi}$ is the pion decay constant and $e$ is the inverse strength parameter of the quartic stabilizing term. The term $\mathcal{L}_{S B}$ accounts for the chiral symmetry breaking terms responsible for the different pseudoscalar meson masses and decay constants. Its explicit form can be found in e.g. ref. [19]. The parameter $x$ determines the strength of the coupling $1-x$ of the $\eta$ to baryonic matter. This may be determined by the empirical value of the real part of the $\eta$-nucleon scattering length $a_{\eta N}=0.717 \pm 0.030$ [20] (this value is close to that in ref.[21]).

The effective Lagrangian for the $\eta$-skyrmion system can be obtained using the usual ansatz:

$$
U=U_{\pi} e^{i \lambda_{8} \eta / f_{\eta}}
$$

where $U_{\pi}$ represents the $\mathrm{SU}(2)$ skyrmion field and $\eta$ the field of the $\eta$-meson. For $U_{\pi}$ we shall employ the rational map ansatz of ref. [4], which provides a satisfactory description of the minimum energy configurations of baryons with $B>1$. Thus

$$
U_{\pi}=e^{i \vec{\tau} \cdot \hat{\pi}_{n} F(r)}
$$

where $F(r)$ is the chiral angle, which depends on the distance to the center of the skyrmion, and $\hat{\pi}$ is defined as the vector field

$$
\hat{\pi}_{n}=\frac{1}{1+\left|R_{n}(z)\right|^{2}}\left(2 \operatorname{Re}\left[R_{n}(z)\right], 2 \operatorname{Im}\left[R_{n}(z)\right], 1-\left|R_{n}(z)\right|^{2}\right) .
$$

Here $R_{n}(z)$ is the rational map for winding (baryon) number $B=n$, and the variable $z$ is defined terms of the usual spherical coordinates $\theta, \varphi$ as $z \equiv \tan (\theta / 2) \exp (i \varphi)$. For $n=1$, $R(z)=z$ and (3) reduces to Skyrme's hedgehog ansatz. The explicit forms of the rational maps for various values of $n$ are given in ref. [⿴囗十). In the case of the $\alpha$-particle, for which $n=4$, the rational map takes the form

$$
R_{4}(z)=\frac{z^{4}+2 \sqrt{3} i z^{2}+1}{z^{4}-2 \sqrt{3} i z^{2}+1} .
$$

Insertion of the ansatz (3),(4) into the Lagrangian density of the model yields, upon expansion of (2) to second order in the $\eta$ field, the following wave equation for the $\eta$-meson in the skyrmion field:

$$
\left[\frac{1}{r^{2}} \frac{\partial}{\partial r}\left(r^{2} \beta \frac{\partial}{\partial r}\right)+\alpha \omega_{\eta}^{2}-\gamma^{2}\right] \eta(r)=0 .
$$


where the $\eta$ meson has been assumed to be in an $S$-state and the auxiliary functions $\alpha, \beta, \gamma$ have been defined as

$$
\begin{aligned}
\alpha & =1+\frac{1-x}{e^{2} f_{\eta}^{2}}\left[F^{\prime 2}+2 n \frac{\sin ^{2} F}{r^{2}}\right], \\
\beta & =1+2 n \frac{1-x}{e^{2} f_{\eta}^{2}} \frac{\sin ^{2} F}{r^{2}} \\
\gamma^{2} & =\frac{4 f_{K}^{2}}{3 f_{\eta}^{2}} m_{K}^{2}-\frac{m_{\pi}^{2} f_{\pi}^{2}}{3 f_{\eta}^{2}}(2-\cos F) .
\end{aligned}
$$

Here, $\omega_{\eta}$ is the energy of the $\eta$-meson. The differential equation (5) admits numerical solution for any $n$, once the chiral angle $F(r)$ has been obtained by minimizing the corresponding multiskyrmion mass.

In our numerical calculations the following 3 sets of parameter values will be used: (1) $f_{\pi}=64.5 \mathrm{MeV}, e=5.45, m_{\pi}=0$, (2) $f_{\pi}=54 \mathrm{MeV}, e=4.84, m_{\pi}=138$, (3) $f_{\pi}=93$ $\mathrm{MeV}, e=4.25, m_{\pi}=138$. The parameter sets (1) and (2) reproduce the empirical values of the nucleon and $\Delta(1232)$ masses [6], whereas set (3), which uses the empirical value for the pion decay constant $f_{\pi}$, reproduces the empirical $N \Delta(1232)$ mass splitting, but overpredicts their absolute masses. The kaon mass is taken at its empirical value $m_{K}=495 \mathrm{MeV}$ and the $\eta$ decay constant $f_{\eta}$ is determined so that $f_{\eta} / f_{\pi}=1.28$, which ratio is consistent with the empirical ratio 1.22 between the kaon and pion decay constants [22].

The parameter $x$, which determines the strength of the $\eta$-skyrmion coupling $1-x$ is determined so that the continuum solution of (5) for $n=1$ yields the empirical value for the real part of the $\eta-N$ scattering length. With the parameter sets (1), (2) and (3) above this value is obtained with $x=0.92,0.96$ and 0.89 respectively. Given the value for $x$ the wave equation (5) may be solved for increasing baryon number $n=A$ to test for the existence of $\eta$-nucleus bound states.

In Table 1 we show the predicted binding energies for $A=3-5$. While no bound state is formed by $\eta$-mesons and deuterons (in conformity with experiment), all parameter sets lead to the existence of weakly bound states of the $\eta$-mesons and $\alpha$-particles, with binding energies that vary from $30 \mathrm{KeV}$ to $1.3 \mathrm{MeV}$. With the parameter set (3) a bound state is also obtained for ${ }^{3} \mathrm{He}$, but as the nucleon and $\Delta(1232)$ masses are overestimated by several hundred $\mathrm{MeV}$ with that parameter set, unless quantum corrections are taken into account, this prediction appears as less reliable. Consequently the binding energy estimate of $30 \mathrm{KeV}$ for the $\eta-\alpha$-particle state also appears more realistic than the value $1.3 \mathrm{MeV}$ obtained with parameter set 3 . In any case, the conclusion that there will be a bound state in the case of the $\alpha$-particle seems to be robust in view of the fact that it appears for a wide range of parameter values.

One may speculate whether the $\eta$-meson coupling to baryonic matter is strong enough to produce a bound Borromean system of $\eta$-mesons and the otherwise unbound ${ }^{5}$ He. A direct application of the present method of calculation would lead to a binding energy of the $\eta$-meson to the 5 -nucleon system of about $1 \mathrm{MeV}$ (see Table 1 ).

The main conclusion is then that the $\eta$-meson should form a bound state with the $\alpha$-particle, with a binding energy of about $30 \mathrm{KeV}$. This prediction conforms with recent experimental information on the $\eta$-nuclear interaction [11, 12, 20]. Precision experiments 
at cooler ring facilities are expected to settle the issue in the near future.

This work was initiated while the authors were participating at the joint JLAB-ECT* workshop on "N* and nonpertubative QCD" at the ECT* in Trento. We thank the organizers of the workshop and the staff of the ECT* for their hospitality during that period. The work of NNS is partially supported by grants of Fundación Antorchas and ANPCyT, Argentina and that of DOR by the Academy of Finland under contract 34081.

\section{References}

1. T. H. R. Skyrme, Proc. Roy. Soc. A260 (1961) 127.

2. E. Braaten, S. Townsend and L. Carson, Phys. Lett, B235 (1990) 147.

3. R. A. Battye and P. M. Sutcliffe, Phys. Rev. Lett. 79 (1997) 363.

4. C. J. Houghton, N. S. Manton and P. M. Sutcliffe, Nucl. Phys. B510 (1998) 507.

5. P. Irwin, eprint hep-th/9804149

6. I. Zahed and G. E. Brown, Phys. Rept. 142 (1986) 1.

7. E. M. Nyman and D. O. Riska, Rept. Prog. Phys. 53 (1990) 1137.

8. M. Schvellinger and N.N. Scoccola, Phys. Lett. B430 (1998) 32.

9. R. S. Bhalerao and L. C. Liu, Phys. Rev. Lett. 54 (1985) 865.

10. Q. Haider and L. C. Liu, Phys. Lett. B172 (1986) 257.

11. H. Calén et al., Phys. Rev. Lett. 80 (1998) 2069.

12. C. Wilkin, Phys. Rev. C47 (1993) R938.

13. N. Willis et al., Phys. Lett. B406 (1997) 14.

14. S. J. Brodsky, I. Schmidt and G. F. de Téramond, Phys. Rev. Lett. 64 (1990) 1011.

15. M. Luke, A. V. Manohar and M. J. Savage, Phys. Lett. B288 (1992) 355.

16. C. Gobbi, D. O. Riska and N. N. Scoccola, Phys. Lett. B296 (1992) 166.

17. E. Witten, Nucl.Phys. B223 (1983) 433.

18. G. Pari, Phys. Lett. B261 (1991) 347.

19. D.O. Riska and N.N. Scoccola, Phys. Lett. B265 (1991) 188. 
20. M. Batinić et al., Physica Scripta 58 (1998) 15.

21. A.M. Green and S. Wycech, Phys. Rev. C55 (1997) R2167.

22. C. Gobbi and N. N. Scoccola, Phys. Lett. B318 (1993) 382.

Table 1. The predicted binding energies $B_{A}$ for nuclei with $A=3,4$ and 5 as obtained for the 3 sets of parameters described in the text.

\begin{tabular}{|c|c|c|c|}
\hline Set & $\begin{array}{c}B_{3} \\
\mathrm{MeV}\end{array}$ & $\begin{array}{c}B_{4} \\
\mathrm{MeV}\end{array}$ & $\begin{array}{c}B_{5} \\
\mathrm{MeV}\end{array}$ \\
\hline 1 & - & 0.03 & 1.1 \\
\hline 2 & - & 0.03 & 0.5 \\
\hline 3 & 0.02 & 1.3 & 3.2 \\
\hline
\end{tabular}

